\title{
On interpolation and sampling in Hilbert spaces of analytic functions
}

\author{
By Bo Berndtsson at Göteborg and Joaquim Ortega Cerdà at Barcelona ${ }^{1}$ )
}

\section{Introduction}

In a series of recent papers Seip [S], Seip-Wallstén [S-W] and Lyubarskii-Seip [L-S] have studied sets of interpolation and sampling for various spaces of analytic functions of one variable. Part of these results concern Hilbert spaces of functions that are square integrable against certain weights, and another, closely related part deals with similar spaces with uniform norms. The methods used in these papers are based on classical-type but intricate constructions of one-variable nature that to some extent go back to Beurling [B].

In [O] Ohsawa has suggested the use of $L^{2}$-techniques for $\delta$ to prove results of the above type. In particular, Ohsawa gives a proof of the sufficiency part of the theorem of Seip-Wallstén concerning interpolation in the space of entire functions in $\mathbb{C}$ satisfying

$$
\int|f|^{2} e^{-|z|^{2}}<\infty
$$

As in the approach initiated by Bombieri, Hörmander and Skoda (see [H]), the main difficulty in such a proof is the construction of a (pluri)-subharmonic function with prescribed singularities at the points where one wishes to interpolate. For this Ohsawa uses part of the constructions of Seip-Wallstén, ultimately going back to Beurling, and he poses as a problem to give a more elementary proof. One purpose of this note is to show how that can be done. As it turns out, the method we use also works for more general weights and therefore also implies the sufficiency part of the theorem by Lyubarskii-Seip. Furthermore, we shall show how the positive direction of the sampling theorem can be obtained in a similar manner, and we shall also permit somewhat more general growth conditions than Lyubarskii-Seip (see Theorem 2).

1) First author supported by the NFR.

Second author partially supported by the DGICYT grant PB92-08084-C02-01 and also by the Comissionat per Universitats i Recerca de la Generalitat de Catalunya. 
In [O] Ohsawa also gives a new proof of the theorem of Seip about interpolation in Bergman spaces of the disk. This part of Ohsawa's work contains two essential ingredients. The first one is, as in the case of entire space, the construction of a subharmonic function with prescribed singularities. In [O] no details of this construction is given, so in section 3 of this paper we shall show how the construction in entire space can be adapted to the disk case. For this we use a so called invariant convolution in the disk, introduced by Ulrich [U]. The other ingredient in Ohsawa's proof is a generalized version of Hörmander's $L^{2}$ estimates for $\bar{\partial}$, inspired by a theorem of Donelly and Fefferman [D-F]. Ohsawa deduces this $L^{2}$-estimate from a more general version involving vector bundles over Kähler manifolds. Since this terminology probably is not so well known among specialists in one complex variable, we shall also take this opportunity to give a direct proof for the disk (see also [Be1] for a related argument). In section 3 we shall also prove the positive part of the sampling theorem, and show that both the interpolation and the sampling theorems of Seip $[\mathrm{S} 2]$ hold for more general weights.

The theorems of Seip et al. also concern spaces that are defined by other norms than $L^{2}$, notably uniform norms. In section 4 we show that the methods of this paper also give results of this type, even in our somewhat more general setting. The general lines of the proofs are the same as in the $L^{2}$ case, with the difference that the $L^{2}$-estimates of Hörmander are replaced by similar estimates in uniform norms from [Be 2] and [Be 3].

One comment is in order. The results of Seip et el. are attractive partly because they are so precise and give necessary and sufficient conditions for interpolation and sampling. This paper gives different proofs of the positive directions of these theorems, but we have no new ideas about proofs of the converse directions. In particular, we do not know if the conditions in Theorem 2 and 4 are also necessary for sampling and interpolation, although this seems likely, and perhaps can be proved along the lines of Beurling [B], and Seip [S], [S2].

\section{Interpolation and sampling in $\mathbb{C}$}

Let $\phi$ be a subharmonic function in $\mathbb{C}$, and let $F_{\phi}^{2}$ be defined by

$$
F_{\phi}^{2}=\left\{h \in H(\mathbb{C}) ;\|f\|_{\phi}^{2}=: \int|f|^{2} e^{-\phi}<\infty\right\} .
$$

By definition, a sequence $\Gamma=\left\{z_{j}\right\} \subset \mathbb{C}$ is sampling for $F_{\phi}^{2}$ if there are constants $A$ and $B$ such that for any $h \in F_{\phi}^{2}$

$$
A\|h\|_{\phi}^{2} \leqq \sum\left|h\left(z_{j}\right)\right|^{2} e^{-\phi\left(z_{j}\right)} \leqq B\|h\|_{\phi}^{2} .
$$

The sequence $\Gamma$ is called interpolating if for any sequence $\left\{c_{j}\right\}$ such that

$$
\sum\left|c_{j}\right|^{2} e^{-\phi\left(z_{j}\right)}<\infty
$$

we can find an $h \in F_{\phi}^{2}$ such that $h\left(z_{j}\right)=c_{j}$. Introducing the notation $l_{\phi}^{2}$ for the space of space of all sequences $\xi=\left\{c_{j}\right\}$ such that $\|\xi\|^{2}=\sum\left|c_{j}\right|^{2} e^{-\phi\left(z_{j}\right)}<\infty$ we see that $\Gamma$ is interpolating iff the natural restriction map from $F_{\phi}^{2}$ to $l_{\phi}^{2}$ is surjective, and sampling iff it is bounded and injective, with closed range. 
In [S] is defined the notion of upper and lower density of a sequence in the following way:

$$
D^{+}(\Gamma)=\limsup _{r \rightarrow \infty} \sup _{z \in \mathbb{C}} n(\Gamma \cap \Delta(z, r)) / \pi r^{2}
$$

and

$$
D^{-}(\Gamma)=\liminf _{r \rightarrow \infty} \inf _{z \in \mathbb{C}} n(\Gamma \cap \Delta(z, r)) / \pi r^{2}
$$

(If $E$ is a set, $n(E)$ denotes the number of elements in $E$.) Finally a sequence is called uniformly separated if the infimum of the distances between distinct points is strictly positive. The main results of Seip [S] and Seip-Wallstén [S-W] are as follows.

Theorem A. A sequence $\Gamma$ is interpolating for $F_{\phi}^{2}$ with $\phi=\alpha|z|^{2}$ if and only if it is uniformly separated and

$$
D^{+}(\Gamma)<\alpha / \pi
$$

Theorem B. A sequence $\Gamma$ is sampling for $F_{\phi}^{2}$ with $\phi=\alpha|z|^{2}$ if and only if it can be written as a finite union of uniformly separated sequences and moreover contains a uniformly separated subsequence $\Gamma^{\prime}$ satisfying

$$
D^{-}\left(\Gamma^{\prime}\right)>\alpha / \pi .
$$

In Lyubarskii-Seip [L-S], an analogous notion of density depending on an angle is introduced and shown to characterize interpolating and sampling sequences for $F_{\phi}^{2}$ when $\phi$ is subharmonic, of class $C^{2}$ and positively homogeneous of degree 2 (i.e. $\phi(t z)=t^{2} \phi(z)$ for $t>0)$.

Unwinding the definitions of $D^{+}$and $D^{-}$we see that $D^{+}<\gamma$ if and only if for some $\delta>0$ and all sufficiently large $r$ and all $z$ it holds that

$$
n(\Gamma \cap \Delta(z, r)) / \pi r^{2}<\gamma-\delta
$$

and that $D^{-}>\gamma$ if and only if for some $\delta>0$ and all sufficiently large $r$ and all $z$ it holds that

$$
n(\Gamma \cap \Delta(z, r)) / \pi r^{2}>\gamma+\delta .
$$

It is not hard to see that both these conditions are equivalent to saying that (1) and (2) hold for some large $r$. For a general subharmonic function $\phi$ we now define a similar notion. Here, as well as in the rest of this paper we let the Laplace operator $\Delta$ be defined as $\Delta=\partial^{2} / \partial z \partial \bar{z}$, a convention which differs from the standard one by a factor 4 .

Definition. The sequence $\Gamma$ is dense with respect to $\Delta \phi$ if for some $r<\infty$ and $\delta>0$ it holds that

$$
n(\Gamma \cap \Delta(z, r)) / r^{2}>\Delta \phi(z)+\delta
$$

for all $z$. 
$\Gamma$ is thin with respect to $\Delta \phi$ if for some $r<\infty$ and $\delta>0$ it holds that

$$
n(\Gamma \cap \Delta(z, r))^{\mu} / r^{2}<\Delta \phi(z)-\delta
$$

for all $z$.

We are now ready to formulate the first version of our main result.

Theorem 1. Suppose $\phi$ is subharmonic in $\mathbb{C}$ and that $\Delta \phi$ is uniformly bounded. Then a uniformly separated sequence $\Gamma$ is

(a) interpolating for $F_{\phi}^{2}$ if $\Gamma$ is thin with respect to $\Delta \phi$,

and

(b) sampling for $F_{\phi}^{2}$ if $\Gamma$ is dense with respect to $\Delta \phi$.

In the light of what we just said it is clear that when $\phi=\alpha|z|^{2}$ this is just a rephrasing of the sufficiency part of the theorem of Seip-Wallstén. Moreover, one can check that when $\phi$ is positively homogeneous of degree 2 we get the result of Lyubarskii-Seip (note that such a function always has a uniformly bounded laplacian if it is of class $C^{2}$ ).

Theorem 1 has as a consequence the following, perhaps more natural, theorem.

Theorem 2. Suppose $\phi$ is subharmonic in $\mathbb{C}$ and that $\Delta \phi$ is uniformly bounded. Then a uniformly separated sequence $\Gamma$ is

(a) interpolating for $F_{\phi}^{2}$ if for some $r<\infty$ and $\delta>0$ it holds that

$$
n(\Gamma \cap \Delta(z, r)) / r^{2}<\frac{1}{\pi r^{2}} \int_{|\zeta-z|<r} \Delta \phi(\zeta)-\delta \text { for all } z
$$

and

(b) sampling for $F_{\phi}^{2}$ if for some $r<\infty$ and $\delta>0$ it holds that

$$
n(\Gamma \cap \Delta(z, r)) / r^{2}>\frac{1}{\pi r^{2}} \int_{|\zeta-z|<r} \Delta \phi(\zeta)+\delta \text { for all } z .
$$

Assume that the condition in Theorem 2(a) (or (b)) holds for a certain value of $r$. Let

$$
\chi_{r}=\frac{1}{\pi r^{2}} \chi_{\Delta(0, r)}
$$

and

$$
\phi_{r}=\phi * \chi_{r}
$$

be the averages of $\phi$ over disks with radius $r . \phi_{r}$ is again subharmonic, and the conditions mean precisely that $\Gamma$ is thin (or dense) with respect to $\Delta \phi_{r}$. From Theorem 1 we conclude that $\Gamma$ is interpolating (sampling) for $F_{\phi_{r}}^{2}$. But it is easily seen that, if the laplacian of 
$\phi$ is uniformly bounded, then $\phi-\phi_{r}=O\left(r^{2}\right)$. Since $r$ is a fixed number, this proves Theorem 2, given Theorem 1.

To prepare for the proof of Theorem 1 let $v=\sum \delta_{z_{j}}$ be the measure consisting of a pointmass at each point in our sequence, which we assume from now on is uniformly separated. Let $E=1 / \pi \log |z|^{2}$ be the fundamental solution of the Laplace operator (with our convention $\Delta E=\delta_{0}$ ). We now define an auxiliary function

$$
v=\left(v-v * \chi_{r}\right) * E .
$$

This function is certainly well defined if $\Gamma$ is finite. Notice that the value of $v$ at $z$ then depends only on the points in $\Gamma$ with $\left|z_{j}-z\right|<r$, since for the other points the two terms in the definition of $v$ cancel by the mean value property for harmonic functions. Therefore we can define $v$ for arbitrary sequences by a limiting procedure. An alternative way to define $v$ is to first let

$$
u_{r}=\left(\chi_{r}-\delta_{0}\right) * E
$$

This function satisfies (and of course is characterized by) the properties

$$
\Delta u_{r}=1 / \pi r^{2}-\delta_{0}
$$

in $|z|<r$, and

$$
u_{r}=\partial u_{r} / \partial n=0
$$

on $|z|=r$. Moreover $u_{r}=0$ in $|z|>r$. Explicitly, $u_{r}$ is given by

$$
u_{r}=\frac{1}{\pi}\left(\frac{|z|^{2}}{r^{2}}-1+\log \left(\frac{r^{2}}{|z|^{2}}\right)\right)
$$

if $|z|<r$ and $u_{r}=0$ otherwise.

In particular $u_{r}$ has compact support so we can define $v$ by

$$
v=-u_{r} * v .
$$

Since $E$ is subharmonic it follows from the submeanvalue property that $u_{r} \geqq 0$ so we always have $v \leqq 0$. Of course it holds that

$$
\Delta v=v-v * \chi_{r} .
$$

Assume now that $\Gamma$ is thin with respect to $\Delta \phi$. This means precisely that for some large enough $r$ and some positive $\delta$

$$
\pi v * \chi_{r}<\Delta \phi-\delta
$$

Let

$$
\psi=\pi v+\phi
$$

Then

$$
\Delta \psi \geqq \pi v+\delta \geqq \delta
$$


$\psi$ satisfies the estimates

(i)

$$
\psi \leqq \phi \quad \text { in } \mathbb{C}
$$

and

$$
|\psi-\log | z-\left.z_{j}\right|^{2}-\phi \mid \leqq C_{r}
$$

in $\left|z-z_{j}\right|<\varepsilon_{0}$, if $\varepsilon_{0}$ is chosen so that $\left|z_{j}-z_{k}\right|>2 \varepsilon_{0}$ for $j \neq k$. (ii) just says that

$$
|\pi v-\log | z-\left.z_{j}\right|^{2} \mid \leqq C_{r}
$$

which is clear since $v=-u_{r} * v$ in view of our explicit formula for $u_{r}$.

The proof of Theorem 1 now follows standard lines. Let $\left\{c_{j}\right\}$ be a sequence of values such that

$$
\sum\left|c_{j}\right|^{2} e^{-\phi\left(z_{j}\right)} \leqq A<\infty
$$

The first step in the construction of an interpolating function is to interpolate locally near each point in the sequence $\left\{z_{j}\right\}$. For each $j$ we apply the Riesz decomposition formula in a disk $\Delta_{j}$ of radius $\varepsilon_{0}$ and center $z_{j}$. Write

$$
\phi=h_{j}+G[\Delta \phi]
$$

where $h_{j}$ is harmonic and $G[\Delta \phi]$ is a Green potential. Write $h_{j}=2 \Re H_{j}$, where $H_{j}$ is holomorphic. Let $G_{j}=H_{j}-H_{j}\left(z_{j}\right)$. Then $G_{j}$ is a holomorphic function satisfying

$$
G_{j}\left(z_{j}\right)=0
$$

and

$$
\left|\phi-\phi\left(z_{j}\right)-2 \Re G_{j}\right| \leqq C
$$

in $\Delta_{j}$. This means that

$$
f_{j}=c_{j} e^{G_{j}}
$$

solves

$$
f_{j}\left(z_{j}\right)=c_{j}
$$

and

$$
\left|f_{j}\right|^{2} e^{-\phi} \leqq C\left|c_{j}\right|^{2} e^{-\phi\left(z_{j}\right)}
$$

in $\Delta_{j}$. We next combine the $f_{j}$ 's using a partition of unity.

Let $g \in C_{c}^{\infty}(\mathbb{C})$ be such that $g=1$ for $|z|<\varepsilon_{0} / 2, g=0$ for $|z|>\varepsilon_{0}$ and $|\partial g| \leqq C_{\varepsilon_{0}}$. Put

$$
f(z)=\sum g\left(z-z_{j}\right) f_{j}
$$


Then $f\left(z_{j}\right)=c_{j}$ so $f$ interpolates the right values. Finally we shall modify $f$ to get a holomorphic interpolating function by solving a $\bar{\delta}$-equation. Note first that

$$
|\bar{\partial} f|^{2} e^{-\phi} \leqq C \sum\left|c_{j}\right|^{2} e^{-\phi\left(z_{j}\right)}\left|\bar{\partial} g\left(z-z_{j}\right)\right|^{2} .
$$

We then apply Hörmanders $\bar{\partial}$-theorem $[\mathrm{H}]$, which implies that we can find a solution $U$ to $\bar{\partial} U=\bar{\partial} f$ satisfying

$$
\int|U|^{2} e^{-\psi} \leqq \int|\delta f|^{2} \frac{e^{-\psi}}{\Delta \psi}
$$

Since $\Delta \psi \geqq \delta$ and $\bar{\partial} f$ vanishes when $z \in \Delta\left(z_{j}, \varepsilon_{0} / 2\right)$ for some $z_{j}$ the right hand side is dominated by some constant times

$$
\int|\bar{\partial} f|^{2} e^{-\phi} \leqq C^{\prime} \sum\left|c_{j}\right|^{2} e^{-\phi\left(z_{j}\right)}\left|\bar{\partial} g\left(z-z_{j}\right)\right|^{2} \leqq C^{\prime \prime} A .
$$

Consequently

$$
\int|U|^{2} e^{-\phi} \leqq \int|U|^{2} e^{-\psi} \leqq C^{\prime \prime} A .
$$

Moreover $U\left(z_{j}\right)=0$ for each $z_{j}$ since $e^{-\psi} \sim 1 /\left|z-z_{j}\right|^{2}$ near $z_{j}$. Let

$$
h=f-U \text {. }
$$

Then $h\left(z_{j}\right)=c_{j}$, and $\int|h|^{2} e^{-\phi}<\infty$ since both $f$ and $U$ satisfy this estimate. The proof of Theorem 1 (a) is therefore complete.

We now turn to the sampling part of Theorem 1. For a moment, let $v$ be an arbitrary positive measure on $\mathbb{C}$. Assume $v * \chi_{r} \leqq C$ and that

$$
\pi v * \chi_{r}>\Delta \phi+\delta
$$

which is the analog of the density condition for general measures. Put

$$
v=\left(v-v * \chi_{r}\right) * E
$$

and

$$
\psi=(\pi v+\phi) .
$$

As before $\psi \leqq \phi$ but this time we have

$$
\Delta \psi \leqq v-\delta
$$

Let $h \in F_{\psi}^{2}$, and let $U=|h|^{2} e^{-\psi}$. Since

$$
\Delta \log U \geqq-\Delta \psi
$$

it follows that 
Moreover, $U \in L^{1}(\mathbb{C})$. Let $g$ be a cut-off function such that $g \geqq 0, g=1$ for $|z|<1$ and $g=0$ for $|z|>2$. Then

$$
\lim _{R \rightarrow \infty} \int g(z / R) \Delta U=\lim _{R \rightarrow \infty} \int 1 / R^{2}(\Delta g)(z / R) U=0 .
$$

By the differential inequality for $U$

$$
\int U \Delta \psi \geqq 0
$$

so it follows that

$$
\delta \int|h|^{2} e^{-\psi} \leqq \int|h|^{2} e^{-\psi} d v .
$$

This is already an inequality of sampling type. We would like to choose as before $v=\sum \delta_{j}$ but we cannot do that directly since $\psi$ would then be identically equal to $-\infty$ on the support of $v$, so the inequality would be of no value.

Instead we shall take $v$ to be a smoothed version of a sum of Dirac measures. Let $\Gamma$ be a sequence which is dense with respect to $\Delta \phi$. Let $v$ be defined as

$$
v=t \sum \frac{1}{\pi \varepsilon^{2}} \chi_{\Delta(0, \varepsilon)}\left(z-z_{j}\right)
$$

where $0<t<1$ (we make no distinction between an absolutely continuous measure and its density with respect to Lebesgue measure). Since $\Gamma$ is dense we can choose $t$ so close to 1 that

$$
\pi v * \chi_{r}>\Delta \phi+\delta / 2
$$

for some large $r$. Then $\psi=\pi v+\phi$ will satisfy the estimates

$$
\phi-C_{\varepsilon} \leqq \psi \leqq \phi
$$

and

$$
\left|\psi-t \log \varepsilon^{2}-\phi\right| \leqq C
$$

in $\left|z-z_{j}\right| \leqq \varepsilon$.

Take $h \in F_{\phi}^{2}$. Then $h \in F_{\psi}^{2}$ so (3) holds. This together with (i') and (ii') gives

$$
\delta / 2 \int|h|^{2} e^{-\phi} \leqq C \sum \frac{\varepsilon^{-2 t}}{\varepsilon^{2}} \int_{\left|z-z_{j}\right|<\varepsilon}|h|^{2} e^{-\phi} .
$$

Now we have to use that $h$ is holomorphic (so far we have used only that $\log |h|^{2}$ is subharmonic). Fix $j$ for the moment and write

$$
\int_{\left|z-z_{j}\right|<\varepsilon}|h|^{2} e^{-\phi}=\int_{\left|z-z_{j}\right|<\varepsilon}\left|h e^{-G_{j}}\right|^{2} e^{-\phi} e^{2 \Re G_{j}} \sim \int_{\left|z-z_{j}\right|<\varepsilon}\left|g_{j}\right|^{2} e^{-\phi\left(z_{j}\right)},
$$


where $g_{j}=h e^{-G_{j}}$ is holomorphic in $\left|z-z_{j}\right|<1$ and $g_{j}\left(z_{j}\right)=h\left(z_{j}\right)$. Clearly

$$
\frac{1}{\pi \varepsilon^{2}} \int_{\left|z-z_{j}\right|<\varepsilon}\left|g_{j}\right|^{2} e^{-\phi\left(z_{j}\right)} \leqq 2\left|h\left(z_{j}\right)\right|^{2} e^{-\phi\left(z_{j}\right)}+C \varepsilon^{2} e^{-\phi\left(z_{j}\right)} \sup _{\left|z-z_{j}\right|<\varepsilon}\left|g_{j}^{\prime}\right|^{2} .
$$

But, by Cauchy's estimate

$$
\sup _{\left|z-z_{j}\right|<\varepsilon}\left|g_{j}^{\prime}\right|^{2} e^{-\phi\left(z_{j}\right)} \leqq C \int_{\left|z-z_{j}\right|<1}\left|g_{j}\right|^{2} e^{-\phi\left(z_{j}\right)} \sim \int_{\left|z-z_{j}\right|<1}|h|^{2} e^{-\phi} .
$$

Summing over $j$ we find

$$
\delta / 2 \int|h|^{2} e^{-\phi} \leqq C \varepsilon^{-2 t} \sum\left|h\left(z_{j}\right)\right|^{2} e^{-\phi\left(z_{j}\right)}+C \varepsilon^{2-2 t} \int_{\mathbb{C}}|h|^{2} e^{-\phi} .
$$

Choosing $\varepsilon$ small enough we can absorb the second term on the right in the left hand side. This proves that $\Gamma$ satisfies the left of the inequalities in the sampling condition. The other inequality is trivial since we have assumed that $\Gamma$ is separated, so Theorem $1(\mathrm{~b})$ is now completely proved.

\section{Interpolation and sampling in $\mathbb{D}$}

Let $\phi$ be a subharmonic function in $\mathbb{D}$, and let $F_{\phi}^{2}(\mathbb{D})$ be defined by

$$
F_{\phi}^{2}(\mathbb{D})=\left\{h \in H(\mathbb{D}) ;\|f\|_{\phi}^{2}=: \int_{\mathbb{D}}|f|^{2} \frac{e^{-\phi}}{1-|z|^{2}}<\infty\right\} .
$$

A sequence $\Gamma=\left\{z_{j}\right\} \subset \mathbb{D}$ is sampling for $F_{\phi}^{2}$ if there are constants $A$ and $B$ such that for any $h \in F_{\phi}^{2}(\mathbb{D})$

$$
A\|h\|_{\phi}^{2} \leqq \sum\left|h\left(z_{j}\right)\right|^{2} e^{-\phi\left(z_{j}\right)}\left(1-\left|z_{j}\right|^{2}\right) \leqq B\|h\|_{\phi}^{2} .
$$

The sequence $\Gamma$ is called interpolating if for any sequence $\left\{c_{j}\right\}$ such that

$$
\sum\left|c_{j}\right|^{2} e^{-\phi\left(z_{j}\right)}\left(1-\left|z_{j}\right|^{2}\right)<\infty
$$

we can find an $h \in F_{\phi}^{2}(\mathbb{D})$ such that $h\left(z_{j}\right)=c_{j}$.

A sequence $\Gamma$ is called uniformly discrete or separated if

$$
\inf _{j \neq k} \varrho\left(z_{j}, z_{k}\right)>0,
$$

where $\varrho$ is the pseudo-hyperbolic distance in $\mathbb{D}$,

$$
\varrho(z, \zeta)=\left|\frac{z-\zeta}{1-\bar{z} \zeta}\right| .
$$


Following [S2] we shall define the notion of upper and lower density of a uniformly separated sequence in $\mathbb{D}$. Notice however that our definition differs from the one in [S2] by a factor of two.

$$
D^{+}(\Gamma)=\lim \sup _{r \rightarrow 1} \sup _{\varphi \in \operatorname{Aut}(\mathbb{D})} \frac{\sum_{\substack{z_{n} \in \varphi\left(2_{n}<\left|z_{n}\right|<r \\ \mid\right.}} \log \frac{1}{\left|z_{n}\right|^{2}}}{\log \frac{1}{1-r}}
$$

and

$$
D^{-}(\Gamma)=\liminf _{r \rightarrow 1} \inf _{\varphi \in \operatorname{Aut}(\mathbb{D})} \frac{\sum_{\substack{z_{n} \varepsilon \varphi(\Gamma) \\ 1 / 2<\left|z_{n}\right|<r}} \log \frac{1}{\left|z_{n}\right|^{2}}}{\log \frac{1}{1-r}}
$$

The main results of [S 2] concerning weighted Bergman spaces in the disk are the following:

Theorem C. A sequence $\Gamma$ is interpolating for $F_{\phi}^{2}(\mathbb{D})$ with $\phi=\alpha \log 1 /\left(1-|z|^{2}\right)$ if and only if it is uniformly separated and

$$
D^{+}(\Gamma)<\alpha .
$$

Theorem $\mathbf{D} . \quad A$ sequence $\Gamma$ is sampling for $F_{\phi}^{2}(\mathbb{D})$ with $\phi=\alpha \log 1 /\left(1-|z|^{2}\right)$ if and only if it can be written as a finite union of uniformly separated sequences and moreover contains a uniformly separated subsequence $\Gamma^{\prime}$ satisfying

$$
D^{-}\left(\Gamma^{\prime}\right)>\alpha \text {. }
$$

Let us first reformulate the hypothesis of these theorems. In order to do so we recall the definition (cf. [U], [St]) of the invariant convolution of two functions $f, g$ :

$$
(f * g)(z)=\int_{\mathbb{D}} g\left(\varphi_{z}(\zeta)\right) f(\zeta) d \lambda(\zeta),
$$

where $\varphi_{z}(\zeta)=\frac{z-\zeta}{1-\bar{z} \zeta}$ and $d \lambda(\zeta)$ is the invariant measure, i.e. $d \lambda(z)=d m(z) / \pi\left(1-|z|^{2}\right)^{2}$. Similarly, if $\mu$ is a measure on the disk and $g$ is a function, we define

$$
(\mu * g)(z)=\int_{\mathbb{D}} \frac{g\left(\varphi_{z}(\zeta)\right)}{\pi\left(1-|\zeta|^{2}\right)^{2}} d \mu(\zeta) .
$$

We will need the following easy properties of the invariant convolution. If $f, g, h$ are measurable functions on the disk, and morover $h$ is radial, then

$$
(f * g)=(g * f) \text { and }(f * h) * g=f *(h * g) .
$$

The first property is just a change of variables and the second holds since

$$
h\left(\phi_{z}(\zeta)\right)=h\left(\left|\phi_{z}(\zeta)\right|\right)=h\left(\phi_{\zeta}(z)\right) .
$$


For any $1 / 2<r<1$, we define

$$
\xi_{r}(\zeta)= \begin{cases}0 & \text { if }|\zeta|<1 / 2, \\ \frac{\log \frac{1}{|\zeta|^{2}}}{c_{r}} & \text { if } 1 / 2<|\zeta|<r \\ 0 & \text { if } r<|\zeta|,\end{cases}
$$

where $c_{r}$ is chosen in such a way that $\left\|\xi_{r}\right\|_{L^{1}(d \lambda)}=1$. Observe that $c_{r} / \log \frac{1}{1-r} \rightarrow 1$ as $r \rightarrow 1$, thus $D^{+}(\Gamma)<\gamma$ if and only if there exists some $\delta>0$ such that for all $z \in \mathbb{D}$ and for all $r<1$ large enough it holds

$$
\left(v * \xi_{r}\right)(z)<\gamma-\delta
$$

where $v(z)=\pi \sum_{z_{i} \in \Gamma}\left(1-\left|z_{i}\right|^{2}\right)^{2} \delta_{z_{i}}(z)$. Analogously, $D^{-}(\Gamma)>\gamma$ if and only if there exists some $\delta>0$ such that for all $r<1$ large enough it holds

$$
\left(v * \xi_{r}\right)(z)>\gamma+\delta
$$

We will define now a corresponding notion for arbitrary subharmonic functions. We define the invariant Laplace operator $\tilde{\Delta}$ as $\tilde{\Delta}=\left(1-|z|^{2}\right)^{2} \partial^{2} / \partial z \partial \bar{z}$.

Definition. The sequence $\Gamma$ is dense with respect to $\bar{\Delta} \phi$ if for some $r<1$ and $\delta>0$ it holds that

$$
\left(v * \xi_{r}\right)(z)>\tilde{\Delta} \phi(z)+\delta
$$

for all $z$.

$\Gamma$ is thin with respect to $\tilde{\Delta} \phi$ if for some $r<1$ and $\delta>0$ it holds that

$$
\left(v * \xi_{r}\right)(z)<\tilde{\Delta} \phi(z)-\delta
$$

for all $z$, where as before $v(z)=\pi \sum_{z_{i} \in \Gamma}\left(1-\left|z_{i}\right|^{2}\right)^{2} \delta_{z_{i}}(z)$.

Then the following theorem holds.

Theorem 3. Suppose $\phi$ is subharmonic in $\mathbb{D}$ and of class $C^{2}$. Suppose that $\tilde{\Delta} \phi$ is uniformly bounded. Then a uniformly separated sequence $\Gamma$ is

(a) interpolating for $F_{\phi}^{2}(\mathbb{D})$ if $\Gamma$ is thin with respect to $\tilde{\Delta} \phi$,

and

(b) sampling for $F_{\phi}^{2}(\mathbb{D})$ if $\Gamma$ is dense with respect to $\tilde{\Delta} \phi$.

Just as in section 2, this theorem implies 
Theorem 4. Suppose $\phi$ is subharmonic in $\mathbb{D}$ and of class $C^{2}$. Suppose that $\tilde{\Delta} \phi$ is uniformly bounded. Then a uniformly separated sequence $\Gamma$ is

(a) interpolating for $F_{\phi}^{2}(\mathbb{D})$ if for some $r<1$ and $\delta>0$ it holds that

$$
v * \xi_{r}(z)<\tilde{\Delta} \phi * \xi_{r}(z)-\delta, \quad \forall z \in \mathbb{D},
$$

(b) sampling for $F_{\phi}^{2}(\mathbb{D})$ if for some $r<1$ and $\delta>0$ it holds that

$$
v * \xi_{r}(z)>\tilde{\Delta} \phi * \xi_{r}(z)+\delta, \quad \forall z \in \mathbb{D} .
$$

The proof of Theorem 4, follows the same pattern as the proof of Theorem 2 . Notice that $\tilde{\Delta}\left(\phi * \xi_{r}\right)=\left(\tilde{\Delta} \phi * \xi_{r}\right)$ since $\tilde{\Delta}(\phi(\varphi))=(\tilde{\Delta} \phi) \circ \varphi$, for any $\varphi \in \operatorname{Aut}(\mathbb{D})$.

We now prove Theorem 3. Let $v(z)=\pi \sum_{z_{i} \in \Gamma}\left(1-\left|z_{i}\right|^{2}\right) \delta_{z_{i}}(z)$ and let $E=\log |z|^{2}$. It follows from the definition of invariant convolution that $\tilde{\Delta}(\mu * E)=\mu$ for any measure $\mu$ provided that $\mu * E$ is well defined.

We will need as before an auxiliary function

$$
v=\left(v-v * \xi_{r}\right) * E
$$

Observe that the value of $v(z)$ is determined by the points in $\Gamma$ such that $\varrho\left(z, z_{j}\right)<r$, since $\left(f * \xi_{r}\right)=f$ when $f$ is harmonic. Moreover, since $\xi_{r}$ is radial, we have that

$$
v=v * E-(v * E) * \xi_{r} \leqq 0
$$

That follows from the fact that $v * E$ is subharmonic and $\xi_{r}$ is radial with $\left\|\xi_{r}\right\|_{L^{1}(d \lambda)}=1$. Now define

$$
\psi=\phi+v
$$

Since $v \leqq 0$, we have $\psi(z) \leqq \phi(z)$. Moreover, it follows from the definition that

$$
\left.|v-\log | \frac{z-z_{j}}{1-z \bar{z}_{j}}\right|^{2} \mid \leqq C_{r}
$$

for all $z$ such that $\varrho\left(z, z_{j}\right)<\varepsilon_{0}$, if $\varepsilon_{0}$ is chosen so that $\varrho\left(z_{j}, z_{k}\right)>2 \varepsilon_{0}$ for all $j \neq k$. We are ready to solve the interpolation problem now. Let $\left\{c_{j}\right\}$ be a sequence such that

$$
\sum\left|c_{j}\right|^{2} e^{-\phi\left(z_{j}\right)}\left(1-\left|z_{j}\right|^{2}\right)<+\infty
$$

For each $j$ let $G_{j}$ be a holomorphic function such that $G_{j}\left(z_{j}\right)=0$ and

$$
\left|\phi(z)-\phi\left(z_{j}\right)-2 \Re G_{j}\right| \leqq C,
$$

if $\varrho\left(z, z_{j}\right) \leqq 2 \varepsilon_{0}$. Such a function $G_{j}$ exists since $\tilde{\Delta} \phi$ is uniformly bounded. To see this, we can first apply an automorphism of the disk sending $z_{j}$ to 0 , and then the same proof as in 
section 2 works. Take $g \in C_{c}^{\infty}$ such that $g=1$ for $|z|<\varepsilon_{0} / 2, g=0$ for $|z|>\varepsilon_{0}$ and $|\partial g| \leqq C_{\varepsilon_{0}}$. Set

$$
f(z)=\sum c_{j} g\left(\varphi_{z_{j}}(z)\right) e^{G_{j}} .
$$

This function interpolates the values $c_{j}$ in the points $z_{j}$, and moreover

$$
\int_{\mathbb{Q}}|f|^{2} \frac{e^{-\phi}}{1-|z|^{2}} d m(z) \leqq C \sum\left|c_{j}\right|^{2} e^{-\phi\left(z_{j}\right)}\left(1-\left|z_{j}\right|^{2}\right) .
$$

We finally have to modify the function $f$ to get a holomorphic function. We need the following variant, due to Ohsawa, of Hörmander's theorem.

Theorem $\mathbf{E}$ (Ohsawa). Let $\psi$ be any subharmonic function in the disk such that $\tilde{\Delta} \psi>\delta>0$. Then there is a solution $U$ to the equation $\bar{\partial} U=g$ such that

$$
\int_{\mathbb{D}}|U|^{2} \frac{e^{-\psi}}{1-|z|^{2}} d m(z) \leqq C_{\delta} \int_{\mathbb{D}}|g|^{2} e^{-\psi}\left(1-|z|^{2}\right) d m(z) .
$$

If the sequence $z_{j}$ satisfies the hypothesis in Theorem 4 (a),

$$
\tilde{\Delta} \psi=\tilde{\Delta} \phi+v-v * \xi_{r}>\delta .
$$

Thus, by the above $\bar{\partial}$-theorem, there is a solution to $\bar{\partial} U=\bar{\partial} f$ such that

$$
\int_{\mathbb{\Phi}}|U|^{2} \frac{e^{-\psi}}{1-|z|^{2}} \leqq C \int_{\mathbb{D}}|\bar{\partial} f|^{2} e^{-\psi}\left(1-|z|^{2}\right) .
$$

Since $\bar{\partial} f$ vanishes when $\varrho\left(z, z_{j}\right)<\varepsilon_{0}$ it follows that

$$
|\bar{\partial} f|^{2} e^{-\psi} \leqq C|\bar{\partial} f|^{2} e^{-\phi},
$$

and

$$
\begin{aligned}
|\bar{\partial} f|^{2} e^{-\phi} & \leqq C \sum\left|c_{j}\right|^{2} e^{-\phi+2 \Re G_{j}}\left|\partial g\left(\varphi_{z_{j}}(z)\right)\right|^{2}\left|\varphi_{z_{j}}^{\prime}\right|^{2} \\
& \leqq C \sum\left|c_{j}\right|^{2} e^{-\phi\left(z_{j}\right)}\left|\partial g\left(\varphi_{z_{j}}(z)\right)\right|^{2} \frac{1}{\left(1-\left|z_{j}\right|^{2}\right)^{2}} .
\end{aligned}
$$

Consequently, since

$$
m\left(\varrho\left(z, z_{j}\right)<\varepsilon_{0}\right) \leqq C_{\varepsilon_{0}}\left(1-\left|z_{j}\right|^{2}\right)^{2},
$$

we find

$$
\int_{\mathbb{D}}|U|^{2} \frac{e^{-\phi}}{1-|z|^{2}} \leqq \int_{\mathbb{D}}|U|^{2} \frac{e^{-\psi}}{1-|z|^{2}} \leqq C \sum\left|c_{j}\right|^{2} e^{-\phi\left(z_{j}\right)}\left(1-\left|z_{j}\right|^{2}\right)<+\infty .
$$

Moreover $U\left(z_{j}\right)=0$ since $e^{-\psi} \sim\left|\frac{1-z \bar{z}_{j}}{z-z_{j}}\right|^{2}$ near $z_{j}$. If we then take

$$
h=f-U,
$$


we get that $h\left(z_{j}\right)=c_{j}, h \in H(\mathbb{D})$ and

$$
\int_{\mathbb{D}}|h|^{2} \frac{e^{-\phi}}{1-|z|^{2}}<+\infty .
$$

Hence the interpolation part of theorem 3 is proved.

To prove the sampling part, consider the function

$$
v_{\varepsilon}=\frac{t}{\varepsilon^{2}}\left(\pi \sum\left(1-\left|z_{i}\right|^{2}\right)^{2} \delta_{z_{i}} * \chi_{\Delta(0, \varepsilon)}\right)=\frac{t}{\varepsilon^{2}}\left(v * \chi_{\Delta(0, \varepsilon)}\right)
$$

We can choose $r$ and $t$ close enough to one and $\varepsilon$ small enough so that

$$
v_{\varepsilon} * \xi_{r}>\tilde{\Delta} \phi+\delta / 2
$$

Then consider the functions

$$
v=\left(v_{\varepsilon}-v_{\varepsilon} * \xi_{r}\right) * E
$$

and

$$
\psi=\phi+v
$$

The hypothesis on $v$ from Theorem 4 (b) gives that

$$
\tilde{\Delta} \psi \leqq v_{\varepsilon}-\delta / 2,
$$

and moreover it holds that

$$
\psi \leqq \phi
$$

Furthermore

$$
-C_{\varepsilon} \leqq v \leqq 0,
$$

and one may also verify that

$$
\left|v-t \log \varepsilon^{2}\right| \leqq C,
$$

for any $z$ such that $\varrho\left(z, z_{j}\right)<\varepsilon$.

Let $h \in F_{\phi}^{2}(\mathbb{D})$, and put $U=|h|^{2} e^{-\psi}$. Since $\log |h|$ is subharmonic it follows that

$$
\tilde{\Delta} \log U \geqq-\tilde{\Delta} \psi
$$

and therefore,

$$
\tilde{\Delta} U \geqq-U \tilde{\Delta} \psi
$$

Take a family of cut-off functions $g_{r}$ such that $g_{r} \geqq 0, g=1$ if $|z|<r, g=0$ if

$$
1-|z| \leqq(1-r) / 2
$$


and such that $\tilde{\Delta} g_{r}$ is uniformly bounded by a constant independent of $r$. Then, by (6), $U \in L^{1}\left(\left(1-|z|^{2}\right) d \lambda\right)$ and

$$
\lim _{r \rightarrow 1} \int_{\mathbb{W}}\left(1-|z|^{2}\right) g_{r}(z) \tilde{\Delta} U d \lambda(z)=\lim _{r \rightarrow 1} \int_{\mathbb{\Phi}} \tilde{\Delta}\left(\left(1-|z|^{2}\right) g_{r}(z)\right) U d \lambda(z) \leqq 0 .
$$

Therefore

$$
\lim _{r \rightarrow 1} \int_{\mathbb{D}}\left(1-|z|^{2}\right) g_{r}(z) U \tilde{\Delta} \psi d \lambda \geqq 0
$$

Thus,

$$
\frac{\delta}{2} \int_{\mathbb{W}}|h|^{2} \frac{e^{-\phi}}{\left(1-|z|^{2}\right)} d m(z) \leqq \frac{\delta}{2} \int_{\mathbb{D}}|h|^{2} e^{-\psi} d m(z) \leqq \int_{\mathbb{D}}|h|^{2} e^{-\psi} d v_{\varepsilon}(z) .
$$

Taking into account (7), we obtain

$$
\frac{\delta}{2} \int_{\mathbb{D}}|h|^{2} \frac{e^{-\phi}}{\left(1-|z|^{2}\right)} d m(z) \leqq C \sum_{j} \frac{\varepsilon^{-2 t}}{\varepsilon^{2}} \int_{e\left(z, z_{j}\right)<\varepsilon}|h|^{2} \frac{\mathrm{e}^{-\phi}}{1-\left|z_{j}\right|^{2}} d m(z) .
$$

Consider holomorphic functions $G_{j}$ defined for all $z$ such that $\varrho\left(z, z_{j}\right) \leqq 1 / 2$ with the properties:

$$
G_{j}\left(z_{j}\right)=0 \text { and }\left|\phi(z)-2 \Re G_{j}-\phi\left(z_{j}\right)\right| \leqq C
$$

for all $z$ such that $\varrho\left(z, z_{j}\right) \leqq 1 / 2$. As before, the existence of such functions follows since we have assumed $\tilde{\Delta} \phi$ is bounded. Fix $j$ and call $g_{j}=h e^{-G_{j}}$, then

$$
\int_{\varrho\left(z, z_{j}\right)<\varepsilon}|h|^{2} \frac{e^{-\phi}}{1-\left|z_{j}\right|^{2}}=\int_{\varrho\left(z, z_{j}\right)<\varepsilon}\left|h e^{-G_{j}}\right|^{2} \frac{e^{-\phi}}{1-\left|z_{j}\right|^{2}} e^{2 \Re G_{j}} \sim \int_{\varrho\left(z, z_{j}\right)<\varepsilon}\left|g_{j}\right|^{2} \frac{e^{-\phi\left(z_{j}\right)}}{1-\left|z_{j}\right|^{2}} .
$$

Clearly, since $m\left(\left\{z, \varrho\left(z, z_{j}\right) \leqq \varepsilon\right\}\right) \leqq C \varepsilon^{2}\left(1-\left|z_{j}\right|^{2}\right)^{2}$ it follows that

$$
\begin{gathered}
\frac{1}{\varepsilon^{2}} \int_{e\left(z, z_{j}\right)<\varepsilon}\left|g_{j}\right|^{2} \frac{e^{-\phi\left(z_{j}\right)}}{\left(1-\left|z_{j}\right|^{2}\right)} d m(z) \\
\leqq C\left|h\left(z_{j}\right)\right|^{2} e^{-\phi\left(z_{j}\right)}\left(1-\left|z_{j}\right|^{2}\right)+C \varepsilon^{2} e^{-\phi\left(z_{j}\right)}\left(1-\left|z_{j}\right|^{2}\right)^{3} \sup _{\left|z-z_{j}\right|<\varepsilon}\left|g_{j}^{\prime}\right|^{2} .
\end{gathered}
$$

But, by Cauchy's estimate

$$
\left(1-\left|z_{j}\right|^{2}\right)^{3} \sup _{e\left(z, z_{j}\right)<\varepsilon}\left|g_{j}^{\prime}\right|^{2} e^{-\phi\left(z_{j}\right)} \leqq C \int_{e\left(z, z_{j}\right)<1 / 2}\left|g_{j}\right|^{2} \frac{e^{-\phi\left(z_{j}\right)}}{1-\left|z_{j}\right|^{2}} \sim \int_{e\left(z, z_{j}\right)<1 / 2}|h|^{2} \frac{e^{-\phi}}{1-|z|^{2}} .
$$

Summing over $j$ we find

$$
\frac{\delta}{2} \int_{\mathbb{D}}|h|^{2} \frac{e^{-\phi}}{1-|z|^{2}} \leqq C \varepsilon^{-2 t} \sum\left|h\left(z_{j}\right)\right|^{2} e^{-\phi\left(z_{j}\right)}\left(1-\left|z_{j}\right|^{2}\right)+C \varepsilon^{2-2 t} \int_{\mathbb{D}}|h|^{2} \frac{e^{-\phi}}{1-|z|^{2}} .
$$

If we take $\varepsilon$ small enough, we may absorb the second term on the right in the left hand side, and we obtain the left inequality in the sampling condition. The other inequality is clear since $\Gamma$ is a separated sequence. 


\section{The non-Hilbert case}

The interpolation theorems we have been discussing also have natural analogs in spaces that are not defined by $L^{2}$-norms (see [S1], [S 2] and [S-W]). We shall now briefly indicate how the methods of the previous sections can be adapted to uniform norms.

First we treat the case of entire space. Let, as in section 2, $\phi$ be a subharmonic function with uniformly bounded laplacian in $\mathbb{C}$, and put

$$
B F_{\phi}=\left\{f \in H(\mathbb{C}) ; \sup |f|^{2} e^{-\phi} \leqq C\right\} .
$$

A subsequence $\Gamma$ of $\mathbb{C}$ is interpolating for $B F_{\phi}$ if for any sequence $\left\{c_{j}\right\}$ such that

$$
\sup \left|c_{j}\right|^{2} e^{-\phi\left(z_{j}\right)} \leqq C
$$

there is a function $f$ in $B F_{\phi}$ such that $f\left(z_{j}\right)=c_{j}$.

We then have the following theorem.

Theorem 5. If $\Gamma$ is a separated sequence which satisfies the hypothesis of Theorem 2 (a), then $\Gamma$ is interpolating for $B F_{\phi}$.

To prove this theorem we proceed as with Theorem 1. We take

$$
v(z)=\left(v-v * \chi_{r}\right) * E,
$$

and let

$$
\psi=\phi+v .
$$

Then

$$
\Delta \psi=\Delta \phi+v-v * \chi_{r} \geqq \delta+v .
$$

As in section 2 we first solve the interpolation problem locally. We then find functions $f_{j}$ that are holomorphic in $\Delta_{j}=\Delta\left(z_{j}, \varepsilon_{0}\right)$ satisfying $f_{j}\left(z_{j}\right)=c_{j}$ and

$$
\left|f_{j}\right|^{2} e^{-\phi} \leqq C\left|c_{j}\right|^{2} e^{-\phi\left(z_{j}\right)}
$$

in $\Delta_{j}$. Then put

$$
f(z)=\sum g\left(z-z_{j}\right) f_{j}
$$

where $g$ is the same cut-off function as in section 2. Again, we need to solve a $\bar{\partial}$-equation, but this time we want to estimate the solution in uniform norm. Let

$$
\tilde{\psi}(z)=\sup _{|z-\zeta|<\varepsilon_{0} / 2} \psi(\zeta) .
$$

We apply the following theorem from [Be3]: 
Theorem F. Let $\psi$ be a plurisubharmonic function in $\mathbb{C}^{n}$ such that $i \partial \partial \psi>\delta>0$. Then if $f$ is a $\bar{\partial}$-closed $(0,1)$-form in $\mathbb{C}^{n}$ and $u$ is the solution to $\bar{\partial} u=f$ which is of minimal norm in $L^{2}\left(\mathbb{C}^{n}, e^{-\psi}\right)$, u satisfies

$$
\sup |u| e^{-\tilde{\psi} / 2} \leqq C \sup |f| e^{-\psi / 2} .
$$

This theorem has as a consequence a more precise statement that we will need. Let $h$ be any harmonic function in $\mathbb{C}$ and write $h=\mathfrak{R} H$ where $H$ is entire. Then $v=u e^{-H / 2}$ is the canonical solution to $\bar{\partial} v=e^{-H / 2} f$ in $L^{2}\left(e^{-\psi+h}\right)$. Applying Theorem $\mathrm{F}$ to this situation instead we see that $\tilde{\psi}(z)$ can be replaced by

$$
(\widetilde{\psi-h})(z)+h(z)
$$

where $h$ is any harmonic function in $\mathbb{C}$. It is even enough to assume that $h$ is harmonic in $\Delta_{z}=: \Delta\left(z, \varepsilon_{0} / 2\right)$, since any such $h$ can be approximated by functions that are globally defined. Choosing $h$ to be the harmonic extension of $\psi$ from $\partial \Delta_{z}$ to the interior of $\Delta_{z}$, we see that $\tilde{\psi}(z)$ can be replaced by $\psi(z)-G(z)$ where $G$ is the Green's potential of $\Delta \psi$ over $\Delta_{z}$. In particular, if the laplacian of $\psi$ is uniformly bounded, the Green's potential will be bounded, so Theorem $\mathrm{F}$ holds with $\tilde{\psi}$ replaced by $\psi$.

In our case where $\psi=\phi+v$ one sees in a similar way that we can replace $\tilde{\psi}$ by $\phi$ in $(*)$.

Let us now for a moment suppose that our sequence $\Gamma$ is finite. Then $\partial f$ lies in $L^{2}$ so Theorem $\mathrm{F}$ applies and we see that the canonical solution to $\bar{\partial} u=\bar{\partial} f$ satisfies

$$
\sup |u|^{2} e^{-\phi} \leqq C \sup |\bar{\partial} f|^{2} e^{-\psi / 2} \leqq C \sup \left|c_{j}\right|^{2} e^{-\phi} .
$$

Moreover the canonical solution also satisfies the $L^{2}$ estimate from section 2, so it follows that $u\left(z_{j}\right)=0$. Letting $h=f-u$ we get an interpolating function in $B F_{\phi}$. Since the norm does not depend on the number of points in the sequence, we can also permit infinite sequences by a normal family argument.

We can also treat the disk case in a similar manner. Let $\phi$ be subharmonic in the disk, and suppose that the invariant laplacian of $\phi$ is uniformly bounded. Put

$$
B F_{\phi}(\mathbb{D})=\left\{f \in H(\mathbb{D}) ; \sup _{z \in \mathbb{D}}|f(z)|^{2} e^{-\phi} \leqq C\right\} .
$$

A subsequence $\Gamma$ of the disk is interpolating for $B F_{\phi}$ if for any sequence $\left\{c_{j}\right\}$ such that

$$
\sup \left|c_{j}\right|^{2} e^{-\phi\left(z_{j}\right)}\left(1-\left|z_{j}\right|^{2}\right) \leqq C
$$

there is a function $f$ in $B F_{\phi}$ such that $f\left(z_{j}\right)=c_{j}$. We then have

Theorem 6. Let $\Gamma$ be a sequence in the disk which satisfies the hypothesis of Theorem 4 (a). Then $\Gamma$ is interpolating for $B F_{\phi}(\mathbb{D})$.

The proof again follows the same pattern as in the $L^{2}$ case; the only difference being that we need to replace the $L^{2}$-estimates for $\bar{\partial}$ by uniform estimates. $\operatorname{In}[\mathrm{Be} 3]$ there is also a 
theorem analogous to Theorem $\mathrm{F}$ for the case of the disk (or ball in $\mathbb{C}^{n}$ ) but it requires that $\tilde{\Delta} \phi>4$. In one dimension one can avoid this restriction by instead appealing to the following theorem from $[\mathrm{Be} 2]$.

Theorem G. Let $\psi$ be a subharmonic function and

$$
\eta=: \min ((1-|z|) \Delta \psi, 1 /(1-|z|)) .
$$

Let $f$ be a function in $\mathbb{D}$ such that

$$
\sup \frac{|f|}{\eta} e^{-\psi / 2}<+\infty
$$

Let $u$ be the canonical solution to $\bar{\partial} u=f$ in $L_{\psi}^{2}$. Then

$$
\sup |u| e^{-\bar{\psi} / 2} \leqq C \sup \frac{|f|}{\eta} e^{-\psi / 2}
$$

where $\tilde{\psi}(z)=\sup _{\varrho(z, \zeta)<1 / 2} \psi(\zeta)$.

In our construction from section 3 it holds (after subtraction of a harmonic function like in the case of entire space), that $\tilde{\psi}-\phi$ is uniformly bounded, and moreover the corresponding $\eta$ will be of size $1 /\left(1-|z|^{2}\right)$. Therefore Theorem 6 follows in the same way as Theorem 5 .

Finally, it may be worth remarking that since we use the canonical solution of the $\bar{\gamma}$ equation, we can interpolate between $L^{2}$ and $L^{\infty}$ and get similar results in $L^{p}$ for $p$ between 2 and infinity.

\section{Appendix. A proof of Theorem E}

The one dimensional case of Hörmander's theorem that we used in section 2 says that if $\phi$ is subharmonic in a domain in $\mathbb{C}$ we can solve the equation $\bar{\partial} u=g$ with the estimate

$$
\int|u|^{2} e^{-\phi} \leqq \int \frac{|g|^{2}}{\Delta \phi} e^{-\phi}
$$

in $\Omega$.

That $\bar{\partial} u=g$ in the sense of distributions means that if $\alpha$ is any function in $C_{c}^{\infty}(\Omega)$ it holds that

$$
\int g \cdot \bar{\alpha}=-\int u \frac{\overline{\partial \alpha}}{\partial z}
$$
Taking the supremum of the norm of the right hand side over all $g$ such that $\int \frac{|g|^{2}}{\Delta \phi} e^{-\phi} \leqq 1$,
we get from (8)

$$
\int \Delta \phi|\alpha|^{2} e^{\phi} \leqq \int\left|\frac{\partial \alpha}{\partial z}\right|^{2} e^{\phi}
$$


for all $\alpha \in C_{c}^{\infty}(\Omega)$. Conversely (9) implies Hörmander's theorem, and the usual proof of (8) consists in establishing (9) using integration by parts.

To prove Theorem $\mathrm{E}$ we let $\Omega=\mathbb{D}$ be the unit disk and put as before

$$
\phi_{0}=\log 1 /\left(1-|z|^{2}\right)
$$

Put $\phi=\psi+\phi_{0}$ in (9). The crucial point is that $\phi_{0}$ satisfies

$$
\Delta \phi_{0} \geqq\left|\partial \phi_{0}\right|^{2} \text {. }
$$

Substitute $\alpha=\gamma e^{-\phi_{0}}$ in (9), and assume $\Delta \psi \geqq \delta \Delta \phi_{0}$. Then we get

$$
\begin{gathered}
(\delta+1) \int \Delta \phi_{0}|\gamma|^{2} e^{\psi-\phi_{0}} \leqq \int\left|\frac{\partial \gamma}{\partial z}-\gamma \frac{\partial \phi_{0}}{\partial z}\right|^{2} e^{\psi-\phi_{0}} \\
\leqq(1+2 / \delta) \int\left|\frac{\partial \gamma}{\partial z}\right|^{2} e^{\psi-\phi_{0}}+(1+\delta / 2) \int|\gamma|^{2}\left|\frac{\partial \phi_{0}}{\partial z}\right|^{2} e^{\psi-\phi_{0}} .
\end{gathered}
$$

Rearranging and using (10) we obtain

$$
(\delta / 2) \int \Delta \phi_{0}|\gamma|^{2} e^{\psi-\phi_{0}} \leqq(1+2 / \delta) \int\left|\frac{\partial \gamma}{\partial z}\right|^{2} e^{\psi-\phi_{0}},
$$

or more explicitly

$$
\int|\gamma|^{2} e^{\psi} /\left(1-|z|^{2}\right) \leqq C / \delta^{2} \int\left|\frac{\partial \gamma}{\partial z}\right|^{2}\left(1-|z|^{2}\right) e^{\psi}
$$

A standard functional analysis argument then shows that for a given $g$ we may find $u$ such that

$$
\int g \cdot \bar{\gamma}=-\int u \cdot \frac{\overline{\partial \gamma}}{\partial z}
$$

for all $\gamma \in C_{c}^{\infty}(\mathbb{D})$, and

$$
\int|u|^{2} \frac{e^{-\psi}}{1-|z|^{2}} \leqq C_{\delta} \int|g|^{2} e^{-\psi}\left(1-|z|^{2}\right) .
$$

This completes the proof of Theorem E.

Acknowledgement. Part of this work was done while the second author was visiting the Math. Department at Göteborg. He wants to express his heartfelt gratitude to the institution for its invitation and to its members of their hospitality.

\section{References}

[Be 1] B. Berndtsson, A simple proof of an $L^{2}$-estimate for $\partial$ on complete Kähler manifolds, Report, 1992.

[Be 2] B. Berndtsson, Weighted estimates for $\partial$ in C, Duke Math. J. 66 (1992), 239-255.

[Be3] B. Berndtsson, Uniform estimates with weights for the $\partial$-equation, J. Geom. Anal., to appear. 
[B] A. Beurling, The collected works of Arne Beurling Vol. 2, Birkhäuser, Boston (1989), 341-365.

[D-F] H. Donelly and C. Fefferman, $L^{2}$-cohomology and index theorem for the Bergman metric, Ann. Math. 118 (1983), 593-618.

[H] L. Hörmander, An introduction to complex analysis in several variables, 3rd edition, Van Nostrand (1990), p. 96.

[L-S] Y. Lyubarskii and $K$. Seip, Sampling and interpolation of entire functions and exponential systems in convex domains, Ark. Mat. 32 (1994), 157-193.

[O] T. Ohsawa, On the extension of $L^{2}$-holomorphic functions IV: a new density concept, Preprint, Nagoya University, 1993.

[S] K. Seip, Density theorems for sampling and interpolation in the Bargmann-Fock space I, J. reine angew. Math. 429 (1992), 91-106.

[S2] K. Seip, Beurling type density theorems in the unit disk, Invent. math. 113 (1993), 21-39.

[S-W] K. Seip and R. Wallstén, Density theorems for sampling and interpolation in the Bargmann-Fock space II, J. reine angew. Math. 429 (1992), 107-113.

[Sto] M. Stoll, Invariant potential theory in the unit ball of $\mathbb{C}^{n}$, Cambridge University Press, Cambridge (1994), 31-40.

[U] D. Ulrich, Radial limits of $\mathscr{M}$-subharmonic functions, Trans. Amer. Math. Soc. 292 (1985), 501-518.

\author{
Department of Mathematics, CTH, S-41296 Göteborg, Sweden \\ e-mail: bob@math.chalmers.se \\ Department of Mathematics, UPC (ETSEIB), 08028 Barcelona, Spain \\ e-mail: jortega@ma1.upc.es
}

Eingegangen 6. Dezember 1994 